\title{
41. SPACE DISTRIBUTION OF INTERSTELLAR DUST IN CONNECTION WITH THE GALACTIC SPIRAL STRUCTURE
}

\author{
T. A. URANOVA \\ Moscow University, Moscow, U.S.S.R.
}

\begin{abstract}
A maximum of dust density along the inner edge of the Cygnus arm is found. It seems that the same happens along the inner edges of the Perseus and the Sagittarius arms.
\end{abstract}

Pronik (1962) has examined some large dust clouds, studied by different investigators, and has shown that these clouds form a dust edge near the inner side of the CygnusOrion arm.

The present work deals with the general laws of the distribution of the galactic dark matter in connection with its spiral structure. We have studied the regions of the Milky Way Rift and stretches from $32^{\circ}$ to $54^{\circ}$ and from $67^{\circ}$ to $72^{\circ}$ in longitude, and from $-12^{\circ}$ to $+10^{\circ}$ in latitude. The space distribution of dust was studied with the aid of absorption curves $A_{\mathrm{v}}(r)$. The data of $\mathrm{B}, \mathrm{V}$-photoelectric photometry, taken from 80 works, gave the material needed. Our card catalogue contained about 750 stars, one fourth of them having MK spectral classes; the data of one-dimensional spectral classifications were used after transforming them to the MK system with a careful determination of their systematic discrepancies from the latter. The system of absolute magnitudes and normal colors was taken from Boulon's (1963) work, and the value $R=3.0$ was adopted. The areas studied were divided into regions about $3 \times 3^{\circ}$ in size. The absorption curves were drawn for each region. The selection effect was controlled by a method analogous to that proposed by Neckel (1966). For other directions we have used the data of photographic photometry from Ikhsanov (1959), Metik (1962), Grigorieva (1965; 1969), Mashnauskas (1968), Brodskaja (1956; 1961), Ampel (1959), Adolfsson (1955), Strajzis (1963), Beer (1961), Pronik (1959) and Apriamashvili (1966), after transforming to the B, V, MK-system.

The optical density of the dust $a(r)$, in mag kpc${ }^{-1}$, was determined as the derivative of the absorption curve. Values of $a(r)$, with intervals equal to $50 \mathrm{pc}$, were taken from each curve. These data allowed to study in detail the variations of $a(r)$ with the $z$ coordinate and with the distance in the galactic plane.

The whole thickness of the absorption layer in the solar neighbourhood was estimated $85 \mathrm{pc}$ above the galactic plane and $160 \mathrm{pc}$ below it. This may be both a real fact and the result of the sun's elevation above the galactic plane.

The variations of the dust density along the line of sight were examined for seven directions in the above mentioned sectors; the regions of latitude $|b|=2^{\circ}$ were used. In all directions mentioned the line of sight meets a dense dust front with an absorption of $a(r) \geqslant 5-20 \mathrm{mag} \mathrm{kpc}^{-1}$. The distance of the dust front from the sun increases with the longitude, so that the line of this front is nearly parallel to the edge of the Cygnus arm (Figure 1). 
The results obtained allowed to draw a map of dust density distribution in the galactic plane, with graduations of 1-2 mag $\mathrm{kpc}^{-1}$.

This distribution was compared to the positions of hot objects forming the spiral arms. Figure 2 indicates Morgan's aggregates and a part of Becker's O-B2 clusters. This figure shows that the densest parts of the dust layer are arranged along the inner side of the Cygnus arm both in the western and eastern directions.

In the Sagittarius arm direction the density of dust decreases slowly down to zero. The space transparence between the eastern parts of the Cygnus and Sagittarius arms is traced up to 3-8 $\mathrm{kpc}$ from the sun.

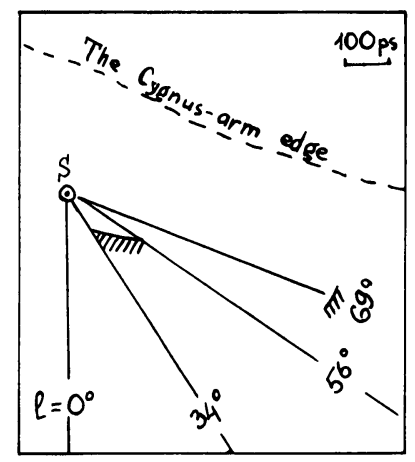

Fig. 1. The position of the dense front of dust in the galactic plane.

One can see more distinctly the concentration of dust between the Cygnus and the Sagittarius arms in cross sections, perpendicular to the arms axes (Figure 3). The $\mathrm{x}$-coordinates in Figure 3 are read out from the inner side of the Cygnus arm to the outer side of the Sagittarius arm; the cross sections refer to distances 200 and $800 \mathrm{pc}$ from the sun. It is seen that the dust is gathered near the edge of the Cygnus arm and its flat extension spreads towards the Sagittarius arm.

Returning to Figure 2 one can see that the central regions of the Cygnus arm as well as of the Sagittarius and Perseus arms, do not contain any dense dust formations.

At the same time, in the interval between the Cygnus and Perseus arms, some dense formations are noticeable near the inner side of the latter, as we can deduce from the absorption curves taken from the work of Brodskaja (1956, 1961), Grigorieva (1965) and Mashnauskas (1968).

The scanty data on dust density beyond the Sagittarius arm suggest the presence of a dust edge on its inner side. (The open clusters absorption curve in the direction $l \sim 310^{\circ}$ indicates a density increase, as well as some individual distant stars with great colour excesses at $l \sim 45^{\circ}$.)

It seems that dust is concentrated at the inner sides of the three known arms of Galaxy, whereas their central parts contain considerably smaller quantities of dust.

Certain discrepancies with the conclusions of Neckel (1966) may be explained in our opinion, both by a more detailed graduation of the dust density assumed in the 




Fig. 2. The distribution of dust in the galactic plane.

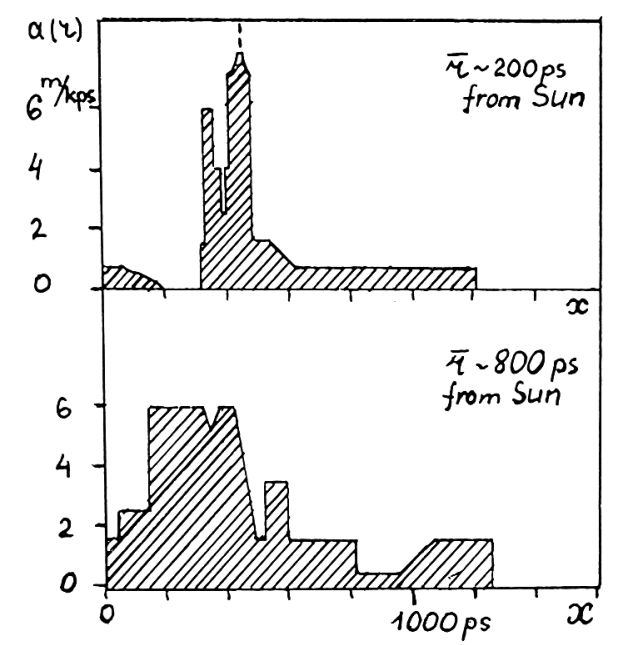

Fig. 3. The distribution of dust density between the Cygnus and the Sagittarius arms (cross sections). 
present work (especially for large values of dust density) and also by the fact that our data are not quite identical.

Detailed results are presented by Uranova $(1968 \mathrm{a}, \mathrm{b})$. The connection of the Milky Way apparent brightness with the distance of the responsible dust matter is also considered there. It is shown that the Milky Way isophotes in the Aquila direction are due to the dust up to $500 \mathrm{pc}$ from the sun.

\section{References}

Adolfsson, T.: 1955, Ark. Astron. 1, No. 34, 495.

Ampel, R.: 1959, Bull. Astron. Obs. Univ. N. Copernicus, Torun, No. 20.

Apriamashvili, S. P.: 1966, Abastumansk. Astrofiz. Obs. Gora Kanobili Bjull. 35.

Beer, A.: 1961, Monthly Notices Roy. Astron. Soc. 123, 191.

Boulon, J.: 1963, J. Observateurs 46, Nos. 10-11.

Brodskaja, E. S. : 1956, Izv. Krymsk. Astrofiz. Obs. 16, 162.

Brodskaja, E. S.: 1961, Izv. Krymsk. Astrofiz. Obs. 26, 382.

Grigorieva, N. B.: 1965, Izv. Krymsk. Astrofiz. Obs. 34, 238.

Grigorieva, N. B.: 1969, Soobshch. Gos. Astron. Inst. P. K. Sternberga, No. 162.

Ikhsanov, R. N.: 1959, Izv. Krymsk. Astrofiz. Obs. 21, 257.

Mashnauskas, I.: 1968, Astron. Obs. Biul. Vilnius No. 22, 47.

Metik, L. P.: 1962, Izv. Krymsk. Astrofiz. Obs. 27, 283.

Neckel, F.: 1966, Z. Astrophys. 63, 221.

Pronik, I. I.: 1959, Izv. Krymsk. Astrofiz. Obs. 21, 268.

Pronik, I. I.: 1962, Astron. Zh. 39, 362.

Strajzis, V.: 1963, Astron. Obs. Biul. Vilnius, No. 8, 18.

Uranova, T. A.: 1968a, Astron. Zh. 45, 1318.

Uranova, T. A.: 1968b, Soobshch. Gos. Astr. Inst. P. K. Sternberga, No. 163. 